\title{
Eficacia de los programas preventivos de trastornos alimentarios en
}

\section{adolescentes: análisis bibliográfico}

\author{
Belén Prieto-Castaño, Juan Carlos Fernández-Méndez, Rosa Marianela Salamea-Nieto y Ma Ángeles González- \\ Fernández \\ Facultad de Ciencias de la Educación, Universidade da Coruña
}

\begin{abstract}
Resumen
En el presente estudio bibliográfico tiene como finalidad realizar una revisión de la literatura sobre la eficacia de programas preventivos de los trastornos alimentarios en población adolescente en el período comprendido entre los años 2010 y 2015. Dicha revisión se llevó a cabo en la base de datos PsycINFO, localizándose un total de 12 trabajos que reunían todos los criterios de la búsqueda. Se concluye que los trabajos revisados, además de poseer un gran rigor metodológico, se muestran eficaces.

Palabras clave: Trastornos alimentarios, anorexia, bulimia, programas de prevención, adolescencia.
\end{abstract}

El objetivo del presente trabajo es realizar un análisis bibliográfico de las investigaciones centradas en programas de prevención de los trastornos alimentarios (TA) en población adolescente, detallando el estado actual de la investigación para conocer las características más relevantes de tales estudios, particularmente la eficacia y los aspectos metodológicos.

Los trastornos alimentarios constituyen un grupo de trastornos psicológicos caracterizados por una conducta alterada ante la ingesta alimentaria (por exceso o por defecto) así como la aparición de comportamientos de control de peso. Los sistemas de clasificación CIE-10 (OMS, 1992) y DSM-5 (APA, 2014) incluyen entre los TA varias categorías entre las que destacan, por sus consecuencias, la anorexia nerviosa (AN) y la bulimia nerviosa $(\mathrm{BN})$.

La AN se define como la pérdida significativa de peso en relación a la altura, ocasionada por la propia persona, mediante la evitación de la ingesta de alimentos que estima le pueden llevar a aumentar de peso, mediante medidas que atentan contra la salud como son vómitos auto-provocados, purgas intestinales, ejercicio excesivo y/o consumo de fármacos; además, se produce una distorsión de la imagen corporal que da lugar a una sobrestimación de su figura o peso corporal. Por su parte, la $\mathrm{BN}$ consiste en una preocupación continua por la comida, con deseos irresistibles de comer, presentando episodios recurrentes de ingesta voraz (atracones) seguidos por conductas compensatorias inapropiadas como el ayuno, el vómito provocado, el abuso de laxantes, diuréticos $u$ otros fármacos adelgazantes o el ejercicio excesivo.

Dichos trastornos aparecen habitualmente durante la adolescencia o en los primeros años de la edad adulta y son más frecuentes en las mujeres; las edades más afectadas son las comprendidas entre los 12 y 24 años (Sancho, Arija, Asorey y Canals, 2007). Estudios epidemiológicos realizados en España en los últimos años ponen de manifiesto que la prevalencia aproximada de estos trastornos, en la población de mayor riesgo, se encuentra en mujeres de 12 a 24 años de edad y se sitúa en su conjunto entre el 4 y el 6,4\%, mientras que en varones la prevalencia se sitúa alrededor del $9-10 \%$ de la encontrada en el género femenino (Peláez, Labrador, y Raich, 2004; RuizLázaro, 2003).

Estos datos son lo suficientemente relevantes para justificar un incremento en la atención política, social y sanitaria dirigida a la prevención de los TA. En este sentido, la Organización Mundial de la Salud (OMS) y la Unión Europea, en sus estrategias de actuación en materia de salud mental, han incluido la promoción de la salud mental y la prevención de los trastornos mentales como objetivos prioritarios (European Commission, 2005; OMS, 2004).

En los países occidentales la prevalencia de los TA va en aumento, así como el importante coste económico derivado del tratamiento de los mismos, poniendo de manifiesto la necesidad de creación de programas preventivos.

\section{Método}

En las últimas décadas se han diseñado un buen número de programas de prevención de las alteraciones del comportamiento alimentario y, por razón, en esta revisión se consideran únicamente programas publicados en el período que comprende de 2010 a 2015, dirigidos a la población adolescente de ambos géneros y que utilizan una metodología rigurosa desde el punto de vista del método científico.

Este análisis cuantitativo bibliográfico ha consistido en la búsqueda de publicaciones que abordan el tema de la prevención de TA en población adolescente y preadolescente a través de una selección informatizada en la base de datos PsycINFO, desarrollada por la American Psychological Association, y que consta de más de 3.000 .000 de registros relacionados principalmente con la Psicología y la Psiquiatría. Las revistas integradas en dicha base de datos deben superar una selección muy rigurosa (Tabla 1).

Los estudios se han localizado bajo los términos Trastornos de la alimentación, anorexia nerviosa, bulimia nerviosa, programas de prevención y adolescencia como palabras clave en los campos de 
búsqueda, realizándose para ambos géneros, en una edad entre 11 y 17 años, así como en todo tipo de publicaciones y con fecha de publicación comprendida entre los años 2010 y 2015. Se descartaron todas las publicaciones que no estaban redactadas en castellano o en inglés.

Tabla 1.

Criterios para la inclusión de revistas en PsycINFO

\begin{tabular}{l} 
Criterios \\
\hline Tiene Comité de Selección \\
Revisión por pares \\
Tener un cuerpo editorial identificable \\
Contener presentaciones originales \\
Cumplimiento de la periodicidad \\
Contener todos los elementos bibliográficos básicos \\
Para revistas electrónicas, identificar un archivo donde se \\
mantengan las copias \\
Tener un ISSN asignado \\
Título, resumen y palabras clave en inglés \\
Demostrar diversidad institucional de revisores y autores \\
(organismos no endogámicos) \\
Contener artículos sustentados con datos empíricos u otros \\
medios
\end{tabular}

\section{Resultados}

Primeramente se ha realizado la búsqueda con cada uno de los términos clave por separado. Las referencias que se han localizado bajo los términos, trastornos de alimentación (eating disorders, ED), anorexia nerviosa (anorexia nervosa, AN), bulimia nerviosa (bulimia nervosa, $\mathrm{BN}$ ) programas de prevención y trastornos alimentarios en adolescencia (eating disorders prevention programs in adolescence, EDPPA) como palabras clave en los campos de búsqueda han sido numerosas, obteniéndose un total de 37.092 para ED, siendo la fecha de la publicación más antigua del año 1734; para AN se encontraron un total de 13.755 (con el año 1905 para la publicación más antigua), mientras que para el término BN se localizaron 10.050, siendo el año 1860 la primera fecha de publicación de dicho tópico y, finalmente, para EDPPA el primer trabajo fechado fue en 1983 y se encontraron 301 resultados. En la Tabla 2 pueden apreciarse el número de revistas científicas, tesis doctorales y libros que se han escrito para cada uno los términos de la búsqueda llevada a cabo.

Tabla 2.

Resultados de la búsqueda en la base de datos

\begin{tabular}{lccc}
\hline Palabra clave & $\begin{array}{c}\text { Revistas } \\
\text { científicas }\end{array}$ & Libros & $\begin{array}{c}\text { Tesis } \\
\text { doctorales }\end{array}$ \\
\hline Eating disorders & 29.468 & 5.033 & 619 \\
Anorexia nervosa & 11.316 & 1.820 & 314 \\
$\begin{array}{l}\text { Bulimia nervosa } \\
\begin{array}{l}\text { Eating disorders prev. } \\
\text { prog. in adolesc. }\end{array}\end{array}$ & 7.621 & 1.429 & 1.000 \\
\hline
\end{tabular}

Dado el gran volumen de trabajos existentes y el interés del presente estudio bibliográfico únicamente se han tenido en cuenta los trabajos que centraban la investigación en los aspectos que relacionaban EDPP en la etapa de la (pre)adolescencia.

De los resultados obtenidos en la presente búsqueda bibliográfica se desprende que existe un gran interés en la comunidad científica por el campo de los ED, AN y $\mathrm{BN}$, si bien es cierto que el número de estudios relacionados con la prevención en la en este período del ciclo vital es mucho menor, localizándose un total de 301 trabajos. Una vez que se aplicaron los criterios planteados en estudio bibliográfico, únicamente 12 trabajos reunían todos los criterios.

Se ha encontrado un trabajo tanto para el año 2010 (Richardson y Paxton) como 2011 (López-Guimerà, Sánchez-Carracedo, Fauquet, Portell y Raich), 3 para el 2013 (Franko, Cousineau, Rodgers y; Golan, Hagay y Tamir; Wilksch y Wade) y 2014 (Berger, Schaefer, Wick, Brix, Bormann, Sowa, Schwartze y Strauss; Rohde, Auslander, Shaw, Raineri, Gau y Stice; Wilksch y Wade) y 4 en el 2015 (Atkinson, M. y Wade; Horney, Stice y Rohde; Weigel, Gumz, Uhlenbusch, Wegscheider, Romer y Löwe; Wilksch, Paxton, Byrne, Austin, McLean, Thompson, Dorairaj y Wade), siendo previsible que puedan publicarse más investigaciones antes de finalizar el año. Una de los primeros aspectos que llaman la atención en la revisión es el gran rigor metodológico en los trabajos (Tablas 3 y 4 ): participantes, instrumentos de evaluación y componentes de los programas preventivos.

Tabla 3.

Características de las muestras

\begin{tabular}{|c|c|c|c|}
\hline Autores & Tamaño muestral & $\begin{array}{c}\text { Edad } \\
\text { media } \\
(\mathrm{DT}) \\
\end{array}$ & Género \\
\hline $\begin{array}{l}\text { Richardson } \\
\text { y Paxton (2010) }\end{array}$ & $\begin{array}{l}194(\mathrm{GE}=104 ; \mathrm{GC}= \\
90)\end{array}$ & $12,4(4,13)$ & $\mathrm{F}$ \\
\hline $\begin{array}{l}\text { López-Guimerà et } \\
\text { al. (2011) }\end{array}$ & $\begin{array}{l}263(\mathrm{GE} 1=57 ; \mathrm{GE} 2= \\
78 ; \mathrm{GC}=128)\end{array}$ & $13.4(0.38)$ & $\mathrm{F}$ \\
\hline $\begin{array}{l}\text { Franko et al. } \\
\text { (2013) }\end{array}$ & $\begin{array}{l}178(\mathrm{GE}=97 ; \mathrm{GC}= \\
81)\end{array}$ & $15,2(0,91)$ & $\mathrm{F}$ \\
\hline $\begin{array}{l}\text { Golan et al. } \\
\text { (2013) }\end{array}$ & $\begin{array}{l}259(\mathrm{GE}=210 ; \mathrm{GC}= \\
49)\end{array}$ & $12-14$ (no) & F y M \\
\hline $\begin{array}{l}\text { Wilksch y Wade } \\
\text { (2013) }\end{array}$ & $\begin{array}{l}115(\mathrm{GE}=51 ; \mathrm{GC}= \\
64)\end{array}$ & $12.7(0,41)$ & F y M \\
\hline $\begin{array}{l}\text { Berger et al. } \\
(2014)\end{array}$ & $\begin{array}{l}533(\mathrm{GE}=188 ; \mathrm{GC}= \\
345)\end{array}$ & 13,1 (no) & F y M \\
\hline $\begin{array}{l}\text { Rohde et al. } \\
\text { (2014) }\end{array}$ & $\begin{array}{l}81(\mathrm{GE}=41 ; \mathrm{GC}= \\
40)\end{array}$ & $12,1(0,9)$ & $\mathrm{F}$ \\
\hline $\begin{array}{l}\text { Wilksch y Wade } \\
\text { (2014) }\end{array}$ & $\begin{array}{l}540(\mathrm{GE}=233 ; \mathrm{GC}= \\
307)\end{array}$ & $13,6(0,37)$ & F y M \\
\hline $\begin{array}{l}\text { Atkinson y Wade } \\
(2015)\end{array}$ & $\begin{array}{l}347(\mathrm{GE} 1=138 ; \mathrm{GE} 2 \\
=108 ; \mathrm{GC}=101)\end{array}$ & $15.7(0.77)$ & $\mathrm{F}$ \\
\hline $\begin{array}{l}\text { Horney et al. } \\
(2015)\end{array}$ & $\begin{array}{l}552(\mathrm{GE}=268 ; \mathrm{GC}= \\
284)\end{array}$ & $16,2(1,4)$ & $\mathrm{F}$ \\
\hline $\begin{array}{l}\text { Weigel et al. } \\
(2015)\end{array}$ & $\begin{array}{l}1848(\mathrm{GE}=\mathrm{sí} ; \mathrm{GC}= \\
\text { sí) }\end{array}$ & 14-17 (no) & $\mathrm{F}$ \\
\hline $\begin{array}{l}\text { Wilksch et al. } \\
(2015)\end{array}$ & $\begin{array}{l}1.316(\mathrm{GE}=\mathrm{sí} ; \mathrm{GC}= \\
\text { sí) }\end{array}$ & $13,2(0,68)$ & F y M \\
\hline
\end{tabular}

En la Tabla 3 pueden verse las características relacionadas con las muestras de participantes. El estudio con mayor tamaño muestral cuenta con un total de 1.848 participantes (Weigel et al., 2015), mientras que 81 es el tamaño de participantes menor (Rohde et 
al., 2014). La menor edad media de los de los sujetos es la del trabajo de Rohde et al. (2014) con 12.1, y la más elevada es de 16,2 (Horney et al., 2015). El 58,3\% (7 investigaciones) de las investigaciones utilizaron participantes de género femenino.

En todos los programas analizados se han empleado instrumentos de evaluación estandarizados de fiabilidad y validez reconocida. Las pruebas psicológicas aplicadas más frecuentemente han sido las siguientes: Eating Disorder Inventory (empleado en 8 investigaciones); Sociocultural Attitudes Towards Appearance Questionnaire (usado en 7 estudios); Dutch Eating Behaviour Questionnaire (5 estudios); Children's Depression Inventory (4 estudios); Eating Disorder Examination-Questionnaire (4 estudios); Eating Attitudes Test (4 estudios); Perceived Sociocultural Pressure Scale (3 estudios).

Todos los programas pusieron de evidencia la eficacia de los programas cuando se compararon con el grupo control. Muchos de los programas incidían se habían diseñado para manejar la insatisfacción corporal y mejorar la autoestima.

Happy Being Me (Richardson y Paxton, 2010) es un breve programa preventivo que se aplica en tres sesiones en la propia clase del alumnado, con la finalidad de incidir en la imagen corporal y en la autoestima, entre otros aspectos. Entre los objetivos se encuentran la reducción de la internalización del ideal de delgadez, la educación de las conversaciones sobre la apariencia y el énfasis las cualidades que no tienen que ver con la apariencia, utilizando diversos procedimientos como actividades de reflexión individual, discusión en clase, visionado de vídeos y actividades de role-play. Hay una mejora en la satisfacción con la imagen corporal y la autoestima en el grupo experimental al ser comparado con el grupo control, tanto en el postratamiento como a los tres meses de seguimiento.

El estudio realizado en España por López-Guimerà et al. (2011) constaba, en uno de los grupos experimentales, de dos componentes: NUT y EM, mientras que en el otro grupo experimental se aplicó únicamente el componente EM. El componente NUT se aplicaba en una sesión de 90 minutos y consistía en educar sobre temas de alimentación y nutrición (por ejemplo, concepto de comida equilibrada, la importancia del agua, análisis de dietas), mientras que el componente EM se desarrollaba en dos sesiones de 90 minutos más dos sesiones de 60 minutos de actividades. Los temas incluidos en las sesiones de 90 minutos eran, entre otros, el ideal femenino de belleza a lo largo de la historia, en las diferentes culturas y en la actualidad en los medios de comunicación, y cómo hacer frente a los mensajes mediáticos. Las sesiones de actividades incluían elementos como el análisis de la publicidad y modelos de cartas de queja a los medios de comunicación por el ideal de belleza presentado. Se apreciaron mayores cambios entre el pre y postintervención para diversas variables en el grupo NUT+EM, en comparación con el grupo control. No se aprecian diferencias significativas entre el grupo que recibió el programa parcial $(\mathrm{EM})$ y el grupo que recibió el programa completo (NUT+ML).
Tabla 4.

Instrumentos utilizados en los diversos programas

\begin{tabular}{ll}
\hline Autores & \multicolumn{1}{c}{ Instrumentos } \\
\hline & Child Depression Inventory, Sociocultural Attitudes \\
& Towards Appearance Questionnaire-3, Eating \\
Richardson & Disorder Inventory: Body Dissatisfaction, Eating \\
y Paxton & Disorder Examination-Questionnaire, Dutch Eating \\
$(2010)$ & Behaviour Questionnaire, EDI: Feelings of \\
& Ineffectiveness, Perceived Socio- cultural Pressure \\
& Scale.
\end{tabular}

López- Body Mass Index, Eating Attitudes Test, Guimerà et al. Questionnaire on influences on body shape model(2011) 26, Indicator of adherence to activities.

Franko et al. Body Mass Index, Eating Disorder Inventory, (2013) Eating Attitudes Test

Golan et al. Sociocultural Attitudes towards Appearance (2013) Questionnaire-3-the media pressure subscale, Contingencies of Self Worth Scale, The Rosenberg Self-Esteem Scale, Eating Attitudes Test-26, Eating Disorders Inventory-2 subscales.

Wilksch y Dutch Eating Behavior Questionnaire-Restrain Wade (2013) scale, Eating Disorder Inventory-Body Dissatisfaction scale, Sociocultural Attitudes Towards Appearance Questionnaire, Children's Depression Inventory-Short Form, Multidimensional Perfectionism Scale, Mcknight Risk Factor Survey, Project Eating Among Teens.

Berger et al. Eating Attitudes Test, German Body Experience (2014) Questionnaire, Sick, Control, One, Fat, Food questionnaire.

Rohde et al. Ideal-Body Stereotype Scale-Revised, The (2014) Perceived Sociocultural Pressure Scale, Satisfaction and Dissatisfaction with Body Parts Scale, Dutch Restrained Eating Scale, Positive Affect and Negative Affect Scale-for Children, Eating Disorder Diagnostic Survey.

Wilkschy Child Depression Inventory e Short Form, Wade (2014) Sociocultural Attitudes Towards Appearance Questionnaire-3, Eating Disorder Inventory: Body Dissatisfaction, Eating Disorder ExaminationQuestionnaire, Dutch Eating Behaviour Questionnaire, EDI: Feelings of Ineffectiveness, Perceived Sociocultural Pressure Scale.

Atkinson y Eating Disorder Examination-Questionnaire, Wade (2015) Positive and Negative Affect Schedule- Expanded, Dutch Eating Behaviour Questio- nnaire-Restraint, Socio-cultural Attitudes To- wards Appearance Scale, Eating Disorder Symptoms, The Clinical Impairment Assessment, The Child and Adolescent Mindfulness Measure.

Horney et al. The Ideal-Body Stereotype Scale-Revised, (2015) Satisfaction and Dissatisfaction with Body Parts Scale, Dutch Restrained Eating Scale, Positive Affect and Negative Affect Scale-Revised, Eating Disorder Diagnostic Interview, DSM-5 Eating Disorders.

Weigel et al. Children's Eating Disorder Examination(2015) Questionnaire, Body Mass Index, Knowledge about ED, Sociocultural Attitudes Towards Appearance Questionnaire, Kid's Eating Disorder Survey, Multidimensional Self Concept Scale, Patient Health Questionnaire, Generalized Anxiety Disorder Scale.

Wilksch et al. Eating Disorder Examination-Questionnaire, Dutch (2015) Eating Behavior Questionnaire, Eating Disorder Inventory, Sociocultural Attitudes Towards Appearance Questionnaire-3, Perceived Sociocultural Pressure Scale, Child Depression Inventory-Short Form, McKnight Risk Factor Survey, Multidimensional Perfectionism Scale: concern over mistakes, Project EAT, Growing Up Today Study. 
El programa BodiMojo (Franko et al., 2013), diseñado para promover una imagen corporal positiva, se administraba durante cuatro períodos de las clases semanales. Las niñas informaron de un aumento de la satisfacción con su aspecto en comparación con los controles; sin embargo, los efectos no se mantuvieron a tres meses de seguimiento. No se encontraron diferencias significativas entre los grupos de intervención y control en los niños.

Golan et al. (2013) diseñaron el programa In Favor of Myself que se administra en ocho sesiones de 90 minutos, una vez a la semana en grupos de 15-20 participantes, y estaba integrado en el curriculum académico. Ofrecía información sobre diversos tópicos relacionados con la imagen corporal, el ideal de belleza, etc. El programa redujo significativamente la brecha entre la figura actual del cuerpo y la figura ideal, así como el impacto del estado de ánimo sobre la autoimagen de las chicas.

Wilksch y Wade (2013) desarrollaron un estudio piloto acerca de Life Smart, un programa de ocho lecciones destinadas a reducir los factores de riesgo tanto para los trastornos alimentarios como para la obesidad. Es un programa psicoeducativo interactivo en el que se abordan en cada una de las lecciones los siguientes temas: salud: hay algo más que tú deberías pensar; salud física: alimentando nuestra salud; salud física: añadiendo descanso y juego a nuestra salud; pensando saludablemente; emociones: ¿qué es lo que hacen por nosotros y cómo podemos manejarlas?; familia y amigos: ¿cómo afectan a nuestra salud?; cómo vivir con inteligencia: ¿qué opinas?, y, finalmente, ¿hacia dónde vamos ? Mirando hacia el futuro. El programa fue útil, y más en las niñas que en los niños, para reducir las preocupaciones sobre el peso y la figura.

Berger et al. (2014) evaluaron los efectos del programa de prevención Torera que se estructuraba en nueve lecciones manualizadas que abordan una amplia gama de problemas relacionados con la alimentación, que abordan temas como las preocupaciones por la comida, la actividad física, las auto-agresiones, aislamiento social, etc. El programa se mostró eficaz para reducir los riesgos de aparición de los TA y fortalecer la autoestima corporal.

El programa MS Body Project (Rohde et al., 2014) tiene como elemento clave la inducción de la disonancia y se desarrolla a lo largo de 6 sesiones semanales de 45 minutos, en grupos de 5 a 8 participantes. Se lleva al participante a tomar una postura actitudinal al criticar el ideal de delgadez mediante la participación verbal, por escrito y mediante ejercicios conductuales, tanto durante las sesiones como en las actividades del hogar. Los participantes del grupo de intervención mostraron reducciones significativas sólo una de las seis variables (la presión de ser delgada), aunque los tamaños del efecto post-test sugirieron reducciones moderadas en riesgo de aparición de síntomas de TA, las reducciones a los 3 meses de seguimiento no se hicieron evidentes.

Wilksch y Wade (2014) diseñaron el programa Media Smart que se aplicaba en el colegio durante 8 sesiones de unos 50 minutos, dos cada semana, y se centra en diversos aspectos de los trastornos alimentarios y los factores de riesgo. El programa permitió conseguir una reducción de los factores de riesgo de TA para los participantes con depresión elevada y una reducción en la tasa de crecimiento para las puntuaciones de riesgo en los participantes con depresión baja.

Atkinson y Wade (2015) pretendían evaluar la viabilidad y eficacia de un programa basado en el mindfulness con la finalidad de reducir el riesgo de aparición de los TA. Los participantes tanto del grupo de intervención centrado en el mindfulness como los participantes del grupo basado en la disonancia recibieron el programa en tres sesiones. Las participantes del grupo que recibieron las sesiones de mindfulness, mostraron reducciones significativas en sus preocupaciones por la percepción del peso y la figura corporal al ser comparadas con las participantes del grupo control. Finalmente, es importante señalar que no se encontraron diferencias al realizar comparaciones con el grupo sometido a la intervención basada en la disonancia cognitiva.

Horney et al. (2015) aplicaron el programa Body Project con la finalidad de reducir los factores de riesgo de aparición de los TA mediante la disonancia, al criticar el ideal de delgadez verbalmente, por escrito y mediante ejercicios conductuales. Se investigaron los predictores de los TA del DSM-5 durante un período de 3 años. El programa se mostró eficaz de cara a reducir el riesgo de aparición de los TA.

Weigel et al. (2015) llevaron a cabo una intervención de 3 sesiones de 90 minutos en dos semanas, y se trabajaban aspectos relacionados con factores de riesgo de los TA tales como el ideal de belleza para chicos y chicas, la imagen corporal, la influencia de los medios, la presión de la familia, etc. El programa contiene elementos interactivos y educativos, y permitió reducir la influencia de factores de riesgo en la aparición de TA.

También en el año 2015 (Wilksch et al., 2015) se compararon varios programas preventivos de TA, que se aplicaban en el colegio: Life Smart, Media Smart y HELP. El primer programa se aplica en ocho lecciones $\mathrm{y}$ se centra en la salud, y contiene elementos relacionados con la alimentación y ejercicio, sueño, estilos de pensamiento y manejo de emociones, y se centra en la prevención de la obesidad. El programa Media Smart y HELPP se centran en la internalización de la información de los medios de comunicación, y la insatisfacción con el cuerpo, así como en los trastornos alimentarios, aplicándose también en 8 sesiones. Los resultados mostraron que las niñas del grupo de Media Smart tenían la mitad de la tasa de aparición de preocupaciones clínicamente significativas relacionadas con la figura y el peso en comparación con las niñas del grupo control a los 12 meses de seguimiento; además, las niñas del grupo Media Smart y el programa Helpp informaron significativamente de menores preocupaciones sobre peso y figura que las niñas del grupo Life Smart a los 12 meses de seguimiento.

\section{Discusión y Conclusiones}

Existe la necesidad de desarrollar programas preventivos de los TA que tengan como objetivo educar 
en los aspectos cruciales de los TA en población adolescente. De acuerdo a Jean Piaget la adolescencia es una etapa que se caracteriza a nivel cognitivo por el desarrollo de un tipo de pensamiento basado en la lógica formal, que genera proposiciones abstractas (Meece, 2000); además, aunque generalmente se desarrolla de una manera estable y continua, las autopercepciones son inestables; la autoconciencia expresa con frecuencia preocupaciones extremas en cómo uno es percibido por los otros.

Los doce artículos revisados son metodológicamente rigurosos: todos contaban con grupos experimental y de control e instrumentos fiables y válidos. En cuanto a los programas preventivos, todos mostraron resultar eficaces para mejorar las variables estudiadas, principalmente la imagen corporal y la autoestima.

Generalmente, los programas que aquí se han analizado contienen elementos educacionales e interactivos, el número de sesiones es reducido: entre dos y nueve, y existen componentes comunes como son la lección magistral, reflexión individual, visionado de vídeos, discusión en grupos y role play. Entre los contenidos más frecuentes están cuestiones de salud como la alimentación, el ideal de belleza actual, el ejercicio, la importancia del pensamiento y el manejo de las emociones.

\section{Referencias}

American Psychiatric Association (2014). DSM-5 Manual Diagnóstico y Estadístico de los Trastornos Mentales. Barcelona: Panamericana.

Atkinson, M. y Wade, T.D. (2015). MindfulnessBased Prevention for Eating Disorders: A SchoolBased Cluster Randomized Controlled Study. International Journal of Eating Disorders. Published online in Wiley Online Library. http://dx.doi.org DOI: 10.1002/eat.22416.

Berger, U., Schaefer, J.M., Wick, K., Brix, C., Bormann, B., Sowa, M., Schwartze, D. y Strauss, B (2014). Effectiveness of Reducing the Risk of Eating-Related Problems Using the German SchoolBased Intervention Program, "Torera", for Preadolescent Boys and Girls. Prevention Science, $15,557-569$.

European Commission (2005) Green Paper. Improving the mental health of the population: Towards a strategy on mental health for the European Union. Bruselas: European Communities. Disponible en http:// ec.europa.eu/health/ph_determinants/life_style/mental/green_paper/mental_gp_en.pdf

Franko, D.L., Cousineau, T.M., Rodgers, R.F. y Roehrig, J.P. (2013). BodiMojo: effective Internetbased promotion of positive body image in adolescent girls. Body Image, 10, 481-488.

Golan, M., Hagay, N. y Tamir S. (2013). The effect of "in favor of myself": preventive program to enhance positive self and body image among adolescents. PLoS One, 8. http://dx.doi.org DOI: 10.1371/journal.pone.0078223.

Horney, A.C., Stice, E. y Rohde, P. (2015). An examination of participants who develop an eating disorder despite completing an eating disorder prevention program: implications for improving the yield of prevention efforts. Prevention Science, 16, 518-526.

López-Guimerà, G., Sánchez-Carracedo, D., Fauquet, J., Portell, M. y Raich, RM. (2011). Impact of a school-based disordered eating prevention program in adolescent girls: general and specific effects depending on adherence to the interactive activities. The Spanish Journal of Psychology, 14, 293-303.

Meece, J. (2000) Desarrollo del niño y del adolescente. Compendio para educadores. México: SEP.

OMS (1992). CIE-10. Trastornos mentales y del comportamiento. Madrid: Meditor.

OMS (2004). Prevention of mental disorders: effective interventions and policy options: summary report. Geneva: World Health Education. Disponible en http://who.int/mental_health/evidence/en/prevention of_mental_disorders_sr.pdf.

Peláez, M.A., Labrador, F.J. y Raich, R.M. (2004). Epidemiología de los trastornos de la conducta alimentaria en España: revisión y estado de la cuestión. Cuadernos de Medicina Psicosomática, 71/72, 33-41.

Richardson, SM y Paxton, SJ. (2010). An evaluation of a body image intervention based on risk factors for body dissatisfaction: a controlled study with adolescent girls. International Journal of Eating Disorders, 43, 112-122.

Rohde, P., Auslander, B.A. Shaw, H, Raineri, K.M.,Gau, J.M. y Eric Stice,M.S. (2014). Dissonance-based prevention of eating disorder risk factors in middle school girls: results from two pilot trials. International Journal of Eating Disorders, 47, 483-494.

Ruiz-Lázaro, P.M. (2003). Epidemiología de los trastornos de la conducta alimentaria en España. Actas Españolas de Psiquiatría, 31, 85-94.

Sancho, C., Arija, M.V., Asorey, O. y Canals, J. (2007). Epidemiology of eating disorders: a two year follow up in an early adolescent school population. European Child \& Adolescent Psychiatry, 16, 495504.

Weigel, A., Gumz, A., Uhlenbusch, N., Wegscheider, K., Romer, G. y Löwe, B. (2015). Preventing eating disorders with an interactive gender-adapted intervention program in schools: Study protocol of a randomized controlled trial. BMC Psychiatry, 15, 21-30.

Wilksch S.M. y Wade T.D. (2013). Life Smart: a pilot study of a school-based program to reduce the risk of both eating disorders and obesity in young adolescent girls and boys. Journal of Pediatric Psychology 38, 1021-1029.

Wilksch, S.M. y Wade, T.D. (2014). Depression as a moderator of benefit from Media Smart: A schoolbased eating disorder prevention program. Behaviour Research and Therapy, 52, 64-71.

Wilksch, S.M., Paxton, S.J., Byrne, S.M., Austin, S.B., McLean, S.A., Thompson, K.M., Dorairaj, K, $y$ Wade, T.D. (2015). Prevention Across the Spectrum: a randomized controlled trial of three programs to reduce risk factors for both eating disorders and obesity. Psychological Medicine, 45, $1811-1823$. 